\title{
Resolvin D1 inhibits endothelial permeability and mitochondrial damage following cardiac ischemia reperfusion in diabetic mice
}

Jialiang Zhang ( $\sim$ zj1094@126.com )

Sichuan University West China Hospital Department of Cardiology https://orcid.org/0000-0001-77370323

\section{Fangyang Huang}

Sichuan University West China Hospital Department of Cardiology

\section{Li Chen}

Sichuan University West China Hospital Department of Cardiology

\section{Guoyong Li}

Sichuan University West China Hospital Department of Cardiology

\section{Dan Xiao}

Sichuan University West China Hospital Department of Cardiology

\section{Wenhua Lei}

Sichuan University West China Hospital Department of Cardiology

Jiahao Zhao

Sichuan University West China Hospital Department of Cardiology

Yanbiao Liao

Sichuan University West China Hospital Department of Cardiology

Changming Li

Sichuan University West China Hospital Department of Cardiology Mao Chen

Sichuan University West China Hospital Department of Cardiology

\section{Research Article}

Keywords: Myocardial ischemia reperfusion injury, RvD1, Mitochondrial damage, Endothelial permeability, Diabetes

Posted Date: November 15th, 2021

DOl: https://doi.org/10.21203/rs.3.rs-593100/v2 
License: (c) (i) This work is licensed under a Creative Commons Attribution 4.0 International License. Read Full License 


\section{Abstract \\ Purpose}

Novel strategies for preventing myocardial ischemia reperfusion injury (MIRI) in a diabetic heart are urgently needed. Resolvin D1 (RvD1) plays important therapeutic roles in inflammatory diseases. However, the therapeutic role of RvD1 in diabetic MIRI is still unknown.

\section{Methods}

Diabetic mice were established with a high-fat diet and streptozotocin (STZ). The mice were pretreated with RvD1 via intraperitoneal injection for 3 days, followed by MIRI. To evaluate the effects of RvD1 on chronic cardiac remodelling, RvD1 was administered for another 2 weeks after MIRI. The effects of RvD1 following MIRI were measured, including the severity of infarct size, regional inflammation, cardiac function, and permeability of cultured endothelial monolayers. Mitochondrial reactive oxygen species (MitoROS) and mitochondrial membrane potential (MMP) were determined using MitoSOX and JC-1.

\section{Results}

RvD1 pretreatment significantly reduced infarct size and the Evans blue content in diabetic injured hearts, which was associated with improved endothelial permeability. At 2 weeks after MIRI, RvD1 treatment partially improved cardiac performance and reduced cardiac fibrosis in diabetic MIRI mice. In vitro, RvD1 attenuated endothelial leakage induced by hypoxia-reoxygenation, $\mathrm{H}_{2} \mathrm{O}_{2}$, and lipopolysaccharide (LPS) under high glucose $(\mathrm{HG})$ conditions. Meanwhile, RvD1 remarkably protected endothelial cells from $\mathrm{H}_{2} \mathrm{O}_{2}-$ induced mitochondrial damage, as evidenced by increased MMP and decreased MitoROS, which was associated with the preservation of VE-cadherin.

\section{Conclusion}

RvD1 alleviates MIRI-induced endothelial permeability and mitochondrial damage injuries in diabetic hearts. Therefore, RvD1 could be a potential therapeutic target for MIRI in diabetes.

\section{Introduction}

Ischemic heart disease, especially acute myocardial infarction, has become a leading cause of morbidity and disability in diabetes[1]. Obesity, hyperglycaemia, and hyperlipidaemia are the most common metabolic disorders identified in diabetes and are also established cardiovascular risk factors[2, 3]. Diabetic hearts are more susceptible to MIRI and patients with diabetes have a higher risk of mortality following reperfusion[4]. Meanwhile, the cardioprotective effects of ischemic and pharmacological 
preconditioning are compromised in the presence of diabetes $[5,6]$. Therefore, new therapeutic strategies to prevent MIRI in diabetic hearts are urgently needed.

Cardiac endothelial cells form the inner cell layer of blood vessels and whereby create a physiological barrier that protects cardiomyocytes against various stressors. The endothelium are also able to finely regulate the oxygen-carbon cycling and nutrient exchange of cardiomyocytes[7]. However, hyperglycaemic environment can induce metabolic disorder and oxidative stress in endothelial cells, which subsequently resulted in inflammatory activation, and damaged permeability[8]. In addition, increased endothelial permeability and excessive cellular swelling represent critical mechanisms of MIRI. Therefore, endothelial cells are becoming a vital target of multiple therapeutic approaches to MIRI in diabetes[9].

Resolvin D1 (RvD1), one member of specialized pro-resolving mediators (SPMs), is considered to possess an important anti-inflammatory capacity $[10,11]$. It was reported that RvD1 was remarkably decreased in plasma samples from patients with diabetes[12]. In addition, it has also been reported that RvD1 decreased infarct size and protected cardiomyocytes in myocardial infarction rats[13]. RvD1 also protects endothelial adherent junction from disruption by inflammatory mediators such as LPS[14]. However, it remains unclear whether RvD1 affects vascular permeability in the condition of hyperglycaemia. Meanwhile, it remains unknown whether the cardioprotective effects of RvD1 were not compromised in diabetic mice after MIRI. In the present study, we sought to investigate the therapeutic effect of RvD1 on cardiac microvascular damage and explored potential protective mechanisms following MIRI in diabetic mice.

\section{Methods And Materials}

\section{Myocardial ischemia and reperfusion injury mice model}

The animal experiments were performed according to the Guide for the Care and Use of Laboratory Animals published by the US National Institutes of Health (NIH Publication No. 85-23, revised 1985). All experimental procedures involving animals were approved by the Ethics Committee of the Animal Care and the Ethics Committee of Sichuan University. For the type 2 diabetic mouse model, 8- to 10-week-old male C57BL/6J mice were fed a high-fat diet for 4 weeks and then given a single intraperitoneal injection of STZ ( $90 \mathrm{mg} / \mathrm{kg}$, Sigma). One week after STZ injection, mice with a 12-hour fasting blood glucose level $\geq 11.1 \mathrm{mmol} / \mathrm{L}$ were considered to have developed diabetes mellitus (DM) [15,16]. For the MIRI model, the left anterior descending (LAD) coronary artery was ligated with a 7-0 suture for 1 hour and then reperfused for 24 hours. For the sham group, a similar surgical procedure was performed, but the LAD coronary artery was not ligated. RvD1 (Cayman Chemical) was administered at a dose of $5 \mu \mathrm{g} / \mathrm{kg} /$ day via intraperitoneal injection.

\section{Echocardiography}


Echocardiography analysis was performed 14 days after MIRI surgery. Mice were anaesthetized with isoflurane, and cardiac function was assessed using a Vevo 3100. Measurements were performed at the midpapillary level from well-aligned M-mode images from the parasternal short axis view.

\section{TTC staining and Evans blue staining}

Following MIRI (1 h ischemia/24 h reperfusion), 2,3,5-triphenyltetrazolium chloride (TTC) (Sigma-Aldrich) and Evans blue (Sigma-Aldrich) dye were applied for measurement of myocardial infarct size. Briefly, at the end of reperfusion, $1 \mathrm{~mL} \mathrm{1 \%}$ Evans blue was injected through the abdominal aorta after re-ligation of the LAD. The heart was then extracted and sectioned once it hardened at $-20^{\circ} \mathrm{C}$, cut into slices $(2-3 \mathrm{~mm})$, and incubated in TTC staining solution for 15 minutes at $37^{\circ} \mathrm{C}$. Subsequently, the slices were fixed in $4 \%$ formaldehyde for 1 hour. Generally, the area stained with Evans blue represented the non-MIRI myocardium, whereas the unstained area was the MIRI myocardium, the red area was at risk, and the white area indicated infarct size. Images were captured using a camera, and the area of the infarcted myocardium as a percentage of the risk area was analysed using ImageJ software.

\section{Vascular permeability analysis}

Vascular permeability was evaluated with Evans blue staining as previously reported[17-19]. In brief, following MIRI, at 3 hours before termination of the experiment, Evans blue dye $(20 \mathrm{mg} / \mathrm{kg})$ was injected intravenously, and the mice were then euthanized and immediately subjected to aorta perfusion with phosphate buffered saline (PBS). The hearts were then excised, and Evans blue dye was eluted in formamide for 18 hours at $70^{\circ} \mathrm{C}$. The absorbance of Evans blue dye at $620 \mathrm{~nm}$ was measured with a Spectra Max 250 spectrophotometer (Molecular Devices, Sunnyvale, CA, USA)

\section{Cell culture}

Human cardiac microvascular endothelial cells (HCMECs) were purchased from Shanghai Huzhen Biotechnology. HCMECs were cultured in DMEM containing $10 \%$ foetal bovine serum and $1 \%$ penicillin/streptomycin in an incubator with $5 \% \mathrm{CO} 2$ at a temperature of $37^{\circ} \mathrm{C}$. The cells were cultured with a final concentration of $30 \mathrm{mM}$ glucose as a HG concentration, and $5.5 \mathrm{mM}$ glucose served as a control. Permeability across endothelial cell monolayers was measured in Transwell units $(0.4 \mu \mathrm{m}$ pore; Corning Costar) under the following conditions in the presence or absence of RvD1 (100 ng/ml): (1) Hypoxic conditions ( $5 \% \mathrm{CO} 2,94 \% \mathrm{~N} 2$, and $1 \%$ O2) used fresh DMEM with $1 \%$ FBS to mimic ischemic conditions for 12 hours, reoxygenation in a 5\% CO2 incubator and DMEM containing $10 \%$ foetal bovine serum and $1 \%$ penicillin/streptomycin for another 12 hours, (2) $\mathrm{H}_{2} \mathrm{O}_{2}(400 \mu \mathrm{M})$ for 12 hours, (3) LPS (500 $\mathrm{ng} / \mathrm{ml})$ for 12 hours. FITC-dextran $(0.5 \mathrm{mg} / \mathrm{ml}$, average molecular mass 40,000 ; Sigma) was added as a fluorescent indicator to the upper chamber for 2 hours before the end experiments. A $100 \mu \mathrm{l}$ sample was taken from the lower chamber, and the fluorescence of FITC-dextran was measured $(485 / 535 \mathrm{~nm}$, absorption/emission wavelengths) with a Spectra Max 250 spectrophotometer (Molecular Devices, Sunnyvale, CA, USA). 


\section{Immunofluorescence}

At the end of the cellular experiments, the medium was removed, and the cells were washed with PBS 3 times, fixed in $4 \%$ paraformaldehyde, and permeabilized with $0.5 \%$ Triton X-100 in PBS. Then, $5 \%$ bovine serum albumin in PBS was used to block cells for $15 \mathrm{~min}$. A primary antibody against VE-cadherin overnight and a secondary antibody for 30 minutes were used for immunostaining. DAPI was used to stain nuclei for 15 minutes, and images were captured with a confocal microscope (N-STORM \& A1, NiKON).

\section{MitosoX staining}

HCMECs were seeded in 24-well plates at a density of $5 \times 10^{4} / \mathrm{ml}$. The cells were cultured with HG, and the $5.5 \mathrm{mM}$ glucose served as a control. The cells were pretreated with RvD1 (100 ng/ml) for 12 hours, followed by $400 \mu \mathrm{M} \mathrm{H}_{2} \mathrm{O}_{2}$ for 4 hours. Then, the cells were washed three times with warm Hank's Balanced Salt Solution (HBSS). MitoSOX-Red (4 $\mu \mathrm{M}$, Thermo Fisher Scientific) dye was added to each well, incubated with the cells for 30 minutes in the dark at $37^{\circ} \mathrm{C}$, and then washed away three times with warm HBSS. Finally, fluorescence intensity was determined using a Celigo imaging cytometer, and images were captured with a confocal microscope (N-STORM \& A1, NiKON).

\section{Mitochondrial transmembrane potential (MMP) assay}

Mitochondrial membrane potential detection was performed using JC-1 (Med Chem Express HY-K0601). According to the manufacturer's instructions, JC-1 $(2 \mu \mathrm{M})$ was added to each well and incubated with the cells at $37^{\circ} \mathrm{C}$ for 20 minutes. The cells were then centrifuged for 3 minutes at $400 \mathrm{~g}$, washed twice with PBS and finally resuspended in $500 \mu \mathrm{L} \mathrm{JC}-1$ fluorescence, which was measured with a flow cytometer.

\section{Western blotting}

HCMECs or heart tissue were lysed using lysis buffer (Beyotime) with protease inhibitors (CST). Protein lysates were separated via SDS-PAGE, transferred to a PVDF membrane, and blotted with antibodies against the target proteins [VE-cadherin (BIOSS), 1:1000 dilution; GAPDH (Affinity Biosciences), 1:1000 dilution], followed by incubation with a goat horseradish peroxidase (HRP)-conjugated antibody (1:10,000 dilution). Bands were detected using an ECL system.

\section{Immunostaining analysis}

For immunostaining of heart sections, hearts were excised from mice after euthanasia, fixed in $4 \%$ paraformaldehyde overnight, embedded in paraffin, and sectioned. The sections were then deparaffinized, rehydrated, retrieved for antigens, and subjected to antigen retrieval. Immunohistochemical analysis of CD45 (Abcam) and CD68 (Abcam) expression was performed, followed by staining with a secondary antibody (goat anti-rabbit antibody). Images were captured with a ZEISS IX83 microscope. For Masson staining, heart sections were deparaffinized, stained with Masson's composite staining solution and a bright-green staining solution and dehydrated in absolute ethanol. Acetic acid was used for washing. 
Finally, the sections were subjected to transparency in xylene and then mounted with neutral gum for analysis. The smooth muscle cell cytoplasm was stained red, and the collagenous fibrous tissue was stained blue by Masson staining. Images were captured using ZEISS IX83.

\section{Quantitative real-time reverse transcription-polymerase chain reaction (RT-PCR)}

Total RNA was extracted from tissues using TRIzol (Invitrogen). After extraction using chloroform and precipitation with isopropanol, the RNA was washed twice with $75 \%$ ethanol, and the RNA concentration was measured after the sample was dissolved in RNase-free water. Subsequently, the RNA was reverse transcribed using a PrimeScript RT Reagent Kit (Takara). RT-PCR assays were performed on a CFX96TM Real-time PCR Detection System (BioRad) using an EvaGreen Supermix Kit (Bio-Rad). Relative mRNA expression was normalized to GAPDH expression. The RT-PCR primer sequences are shown in Table 1.

\section{Transmission electron microscopy (TEM)}

Heart tissues were harvested and quickly fixed in $2.5 \%$ glutaraldehyde for 1 hours, exposed to $1 \%$ osmium tetroxide, sectioned to $60 \mathrm{~nm}$, mounted on Cu grids contrasted with uranyl acetate and lead citrate, and analysed using a JEM-1400 electron microscope.

\section{Statistical analysis}

All experimental data are presented as means \pm SD. Differences between 2 groups of variables were compared using a two-tailed, unpaired t-test. One-way analysis of variance followed by Bonferroni's post hoc test was performed to compare more than three groups. A value of $\mathrm{P}<0.05$ was considered statistically significant.

\section{Results}

\section{RvD1 preconditioning ameliorated MIRI}

After successful establishment of the diabetic mouse model, the mice were pretreated with RvD1 or saline for 3 days. Evans' blue/TTC staining showed that RvD1 significantly reduced the infarct size by $12 \%$ compared with the saline in diabetic mice (Fig. 1A). To study the influence of RvD1 on chronic cardiac remodelling, RvD1 was administered via intraperitoneal injection for another 2 weeks after MIRI. As to cardiac function, MIRI resulted in a significant decline in left ventricular ejection fraction (EF) (Fig. 1B). While, RvD1 administration ameliorated the decline of EF compared with vehicle. Masson trichrome staining showed that RvD1 reduced cardiac fibrosis compared with control at 2 weeks after MIRI in diabetic mice (Fig. 1C, 1D). These observations suggested that RvD1 provided cardioprotective effect in diabetic mice after MIRI.

\section{RvD1 pretreatment inhibited inflammatory responses in diabetic MIRI}


A robust inflammation is a mechanism underlying MIRI. Thus, we evaluated the density of inflammatory cells and gene expression of inflammatory factors in diabetic hearts after MIRI. The immunohistochemical staining showed that there was a robust increase in the density of CD $45^{+}$leukocytes and $\mathrm{CD} 68^{+}$macrophages after MIRI, while the density of inflammatory cells was decreased in RvD1-treated group (Figure 2A-B). Meanwhile, RvD1 significantly downregulated the mRNA expression of IL-1 $\beta$, IL-6 and MCP-1 compared with the vehicle in diabetic MIRI (Fig2C).

\section{RvD1 protected vascular permeability under hyperglycaemia conditions}

As mentioned above, disruption of endothelial barrier function is responsible for MIRI. Thus, we studied whether RvD1 affects vascular permeability after MIRI in diabetic mice. TEM images of the endothelial barrier demonstrated that endothelial cells from diabetic MIRI hearts appeared swollen with increased lysosomal-like inclusions, and the integrity of the endothelial barrier was also damaged, while pretreatment with RvD1 alleviated endothelial barrier damage (Fig. 3A, 3B). Meanwhile, endothelial permeability was measured by assessed Evans blue dye extravasation. As can be seen in Figure 4A-B, RvD1 inhibited the content of Evans blue increased after MIRI in diabetic mice. Together, these data suggest that RvD1 decreased vascular permeability after MIRI in diabetic mice.

Meanwhile, we assess the effect of RvD1 on endothelial permeability using a Transwell device into which a monolayer of HCMECs grew under various stimuli. Dextran-FITC were added to the upper chamber. The leakage of Dextran-FITC in the lower chamber was detected to determine endothelial permeability (Fig. 5A). The fluorescence intensity of FITC in the lower chamber was significantly higher after LPS (Fig. 5B), $\mathrm{H} / \mathrm{R}$ (Fig. 5B) and $\mathrm{H}_{2} \mathrm{O}_{2}$ (Fig. 5C) stimulation, but was attenuated in the presence of RvD1. Taken together, these ex-vivo assays suggested that RvD1 attenuates $\mathrm{H} / \mathrm{R}, \mathrm{H}_{2} \mathrm{O}_{2}$ - and LPS-induced endothelial permeability under $\mathrm{HG}$ conditions.

\section{RvD1-mediated microvascular protection was involved in regulation of mitochondrial function.}

Mitochondrial dysfunction has been considered as one of main driver of endothelial barrier damage after MIRI. MitoROS and mitochondrial transmembrane potential (MMP) are common markers assessing mitochondrial function[20]. Therefore, we firstly examined the level of mitochondrial superoxide in HCMECs. The treatment of $\mathrm{H}_{2} \mathrm{O}_{2}$ and $\mathrm{HG}$ induced a robust production of MitoROS in HCMECs, while RvD1 treatment decreased MitoROS levels. (Fig. 6A, 6B). Next, JC-1 was used to detect the MMP of HCMECs. The flow cytometry analysis revealed that RvD1 pretreatment significantly mitigated the $\mathrm{H}_{2} \mathrm{O}_{2}$-induced mitochondrial membrane potential drop under HG conditions (Fig. 6C). Taken together, RvD1 are able to ameliorate $\mathrm{H}_{2} \mathrm{O}_{2}$-induced mitochondrial dysfunction of endothelial cells under $\mathrm{HG}$ condition.

\section{RvD1 preserved the expression of VE-cadherin after IR in diabetes.}

VE-cadherin-mediated cell-cell adhesion organizes endothelial junctions and maintains barrier function, which is determinant of endothelial permeability. Thus, we investigated the effect of RvD1 on the expression levels of VE-cadherin. As expect, the expression of VE-Cadherin was inhibited by H/R in the 
condition of HG. However, we observed that RvD1 treatment significantly increased the levels of VEcadherin after H/R (Fig. 7A, 7B). Similarly, the in vivo experiments also demonstrated that VE-cadherin was decreased in diabetic heart tissue after MIRI, which was reserved by RvD1 pretreatment (Fig. 6C). This evidence suggests that the effect of RvD1 on endothelial permeability may be mediated by regulating the expression of VE-cadherin.

\section{Discussion}

In the present study, we provide evidence that RvD1 provides cardioprotection against MIRI in diabetic mice by restricting endothelial permeability, effects that were associated with attenuated mitochondrial damage and preservation of VE-cadherin of endothelial cells.

RvD1 has been increasingly accepted as one of principal endogenous anti-inflammatory mediators and thus a focus of therapeutic interest[21, 11, 22, 23]. Liu et al. reported RvD1 reduced infarct size and improved cardiac function in a permanent coronary artery ligation model of rats[13]. The present study extrapolated their findings into MIRI model in diabetic mice. After all, with the development of myocardial reperfusion strategies and the prevalence of metabolic disorders, the MIRI model in diabetic mice provided a more realistic simulation of the clinical practice in the current revascularization era. Of note, previous studies have reported that RvD1 prevents LPS-induced polymorphonuclear leukocyte recruitment to endothelial cells and attenuates endothelial barrier permeability[14, 24, 25]. Here, we observed RvD1 conferred cardioprotection against MIRI in diabetes, which was associated with maintenance of endothelial integrity in diabetic MIRI. Meanwhile, we measured permeability of endothelial monolayer under various conditions, such as hypoxia-reoxygenation, oxidative stress $\left(\mathrm{H}_{2} \mathrm{O}_{2}\right)$, and LPS. Under these conditions, RvD1 partially restrained the degree of hyperpermeability. Collectively, these findings indicate the protective effects of RVD1 on diabetic heart after MIRI is mediated by inhibition of vascular hyperpermeability.

As is well known, mitochondrial function determine the viability and mobility of endothelial cells, thereby influencing the inflammation and endothelial permeability[7]. RvD1 has been proven to reduce oxidative stress, improve mitochondrial morphology and function, and promote mitochondrial DNA repair in primary retinal cells with diabetic retinopathy[26]. Consistent with previous findings, our experiments demonstrated that RVD1 decreased MitoROS levels and protected HCMECs against MMP reduction in condition of $\mathrm{H}_{2} \mathrm{O}_{2}$ as well as $\mathrm{HG}$. VE-cadherin is a component of endothelial cell-to-cell adherens junctions and essential for the barrier function of the vascular endothelium[27]. It has been proven that preservation of VE-cadherin confers cardioprotection in MIRI[17, 18]. Previous study suggested that RvD1 attenuated LPS-induced adherens junction disassembly and endothelial barrier permeability by arresting tyrosine phosphorylation of a-catenin and VE-cadherin[14]. In agreement with previous studies, our results also indicated that RvD1 pretreatment inhibited endothelial monolayer leakage in condition of HG meditated with regulating the expression of VE-cadherin. However, the pathological process of diabetic MIRI is complex and multifactorial. Therefore, further studies are needed to better understand the underlying protective mechanisms of RvD1 in diabetic MIRI. 
In summary, RvD1 ameliorated MIRI in mice via maintaining endothelial integrity with DM, evidenced by a reduction in infarct size and suppression of the inflammatory response. The underlying molecular mechanism was partially through modulation of mitochondrial function and preservation of VE-cadherin. Therefore, RvD1 treatment may be a promising therapeutic drug to protect diabetic hearts against MIRI.

\section{Declarations}

\section{Conflict of Interest}

The authors declare that they have no conflicts of interest.

\section{Ethical Approval}

All applicable international, national, and/or institutional guidelines for the care and use of animals were followed.

\section{Informed Consent}

Informed consent was obtained from all individual participants included in the study.

Author Contributions: MC, CL, FY, and JZ initiated the project and were responsible for the design of the protocol. LY performed the literature review. JZ and FY analysed the data. LC, GL, and WL conducted the animal experiments. JZ, FY, and LC composed the initial draft of the manuscript. JZ, WH, DX, GY, LC, JZ (Jiahao Zhao), and YL were responsible for critical revision of the manuscript and provided important intellectual content. All authors approved the final version of the manuscript submitted for publication.

Funding: This study was supported by The National Natural Science Foundation of China (Grant Nos. 81900258, 8197020724), The Sichuan Science and Technology Program (Grant No. 2020YFS0246), the China Postdoctoral Science Foundation Grant (Grant No. 2019M663521), the Post-Doctor Research Project, Sichuan University (2020SCU12029), and the Post-Doctor Research Project, West China Hospital, Sichuan University (Grant No. 2019HXBH016).

Availability of data and material: The data used to support the findings of this study are transparent and are available from the submitting author (zjl094@126.com) upon reasonable request.

\section{References}

1. Rawshani A, Rawshani A, Franzen S, et al. Risk Factors, Mortality, and Cardiovascular Outcomes in Patients with Type 2 Diabetes. N Engl J Med. 2018;379(7):633-44.

2. Ritchie RH, Abel ED. Basic Mechanisms of Diabetic Heart Disease. Circ Res. 2020;126(11):1501-25.

3. Russo I, Penna C, Musso T, et al. Platelets, diabetes and myocardial ischemia/reperfusion injury. Cardiovasc Diabetol. 2017;16 (1):71. 
4. Dauriz M, Morici N, Gonzini L, et al. Fifteen-Year Trends of Cardiogenic Shock and Mortality in Patients with Diabetes and Acute Coronary Syndromes. Am J Med. 2020;133(3):331-9.

5. Donahoe SM, Stewart GC, McCabe $\mathrm{CH}$, et al. Diabetes and mortality following acute coronary syndromes. JAMA. 2007;298(7):765-75.

6. Penna $\mathrm{C}$, Andreadou I, Aragno M, et al. Effect of hyperglycaemia and diabetes on acute myocardial ischaemia-reperfusion injury and cardioprotection by ischaemic conditioning protocols. $\mathrm{Br} \mathrm{J}$ Pharmacol. 2020;177(23):5312-35.

7. Wang J, Toan S, Zhou H. New insights into the role of mitochondria in cardiac microvascular ischemia/reperfusion injury. Angiogenesis. 2020;23(3):299-314.

8. Takeda Y, Matoba K, Sekiguchi K, et al. Endothelial Dysfunction in Diabetes. Biomedicines. 2020;8(7):182.

9. Herrera-Zelada N, Zuniga-Cuevas U, Ramirez-Reyes A, Lavandero S, Riquelme JA. Targeting the Endothelium to Achieve Cardioprotection. Front Pharmacol. 2021;12:636134.

10. Serhan CN. Pro-resolving lipid mediators are leads for resolution physiology. Nature. 2014;510(7503):92-101.

11. Serhan CN, Chiang N, Van Dyke TE. Resolving inflammation: dual anti-inflammatory and proresolution lipid mediators. Nat Rev Immunol. 2008;8(5):349-61.

12. Xia F, He C, Ren M, Xu FG, Wan JB. Quantitative profiling of eicosanoids derived from n-6 and n-3 polyunsaturated fatty acids by twin derivatization strategy combined with LC-MS/MS in patients with type 2 diabetes mellitus. Anal Chim Acta. 2020;1120:24-35.

13. Liu R, Li Z, Wang Q. Resolvin D1 Attenuates Myocardial Infarction in a Rodent Model with the Participation of the HMGB1 Pathway. Cardiovasc Drugs Ther. 2019;33(4):399-406.

14. Chattopadhyay R, Raghavan S, Rao GN. Resolvin D1 via prevention of ROS-mediated SHP2 inactivation protects endothelial adherens junction integrity and barrier function. Redox Biol. 2017;12:438-55.

15. Ding $M$, Dong Q, Liu Z, et al. Inhibition of dynamin-related protein 1 protects against myocardial ischemia-reperfusion injury in diabetic mice. Cardiovasc Diabetol.2017;16(1):19.

16. Ding $M$, Hu L, Yang H, et al. Reduction of SIRT1 blunts the protective effects of ischemic postconditioning in diabetic mice by impairing the Akt signaling pathway. Biochim Biophys Acta Mol Basis Dis. 2019;1865(6):1677-89.

17. Li Y, Yao Y, Li J, Chen Q, Zhang L, Wang QK. Losartan protects against myocardial ischemia and reperfusion injury via vascular integrity preservation. FASEB J. 2019;33(7):8555-64.

18. Gao XM, Su Y, Moore S, et al. Relaxin mitigates microvascular damage and inflammation following cardiac ischemia-reperfusion. Basic Res Cardiol. 2019;114(4):30.

19. Radu M, Chernoff J. An in vivo assay to test blood vessel permeability. J Vis Exp. 2013;73:e50062.

20. Sivandzade F, Bhalerao A, Cucullo L. Analysis of the Mitochondrial Membrane Potential Using the Cationic JC-1 Dye as a Sensitive Fluorescent Probe. Bio Protoc. 2019;9(1):e3128. 
21. Wang M, Liu M, Zhang J, et al. Resolvin D1 protects against sepsis-induced cardiac injury in mice. Biofactors. 2020;46 (5):766-76.

22. Pope NH, Salmon M, Davis JP, et al. D-series resolvins inhibit murine abdominal aortic aneurysm formation and increase M2 macrophage polarization. FASEB J. 2016;30(12):4192-201.

23. Gerlach BD, Marinello M, Heinz J, et al. Resolvin D1 promotes the targeting and clearance of necroptotic cells. Cell Death Differ. 2020;27(2):525-39.

24. Chattopadhyay R, Mani AM, Singh NK, Rao GN. Resolvin D1 blocks H2O2-mediated inhibitory crosstalk between SHP2 and PP2A and suppresses endothelial-monocyte interactions. Free Radic Biol Med. 2018;117:119-31.

25. Miyahara T, Runge S, Chatterjee A, et al. D-series resolvin attenuates vascular smooth muscle cell activation and neointimal hyperplasia following vascular injury. FASEB J. 2013;27(6):2220-32.

26. Trotta MC, Pieretti G, Petrillo F. et al. Resolvin D1 reduces mitochondrial damage to photoreceptors of primary retinal cells exposed to high glucose. J Cell Physiol. 2020;235 (5):4256-67.

27. Giannotta M, Trani M, Dejana E. VE-cadherin and endothelial adherens junctions: active guardians of vascular integrity. Dev Cell. 2013;26(5):441-54.

\section{Tables}

\begin{tabular}{lcc}
\hline \multicolumn{1}{c}{ Genes } & Forward primer sequence $\left(5^{\prime}-3^{\prime}\right)$ & Reverse primer sequence $\left(5^{\prime}-3^{\prime}\right)$ \\
\hline mGAPDH & ATGGTGAAGGTCGGTGTGACG & GGTCTCGCTCCTGGAAGATGGT \\
m-IL-1 $\beta$ & CTTCAGGCAGGCAGTATC & CAGCAGGTTATCATCATCATC \\
m-MCP-1 & CCACTCACCTGCTGCTACTCA & TGGTGATCCTCTTGTAGCTCTCC \\
m-IL-6 & TGTGCAATGGCAATTCTGAT & GGTACTCCAGAAGACCAGAGGA \\
\hline
\end{tabular}

Table 1. Primers for quantitative RT-PCR.

\section{Figures}


A DM+MIRI

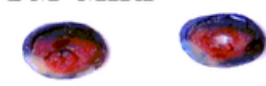

DM+MIRI+RvD1

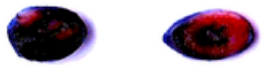

c

B
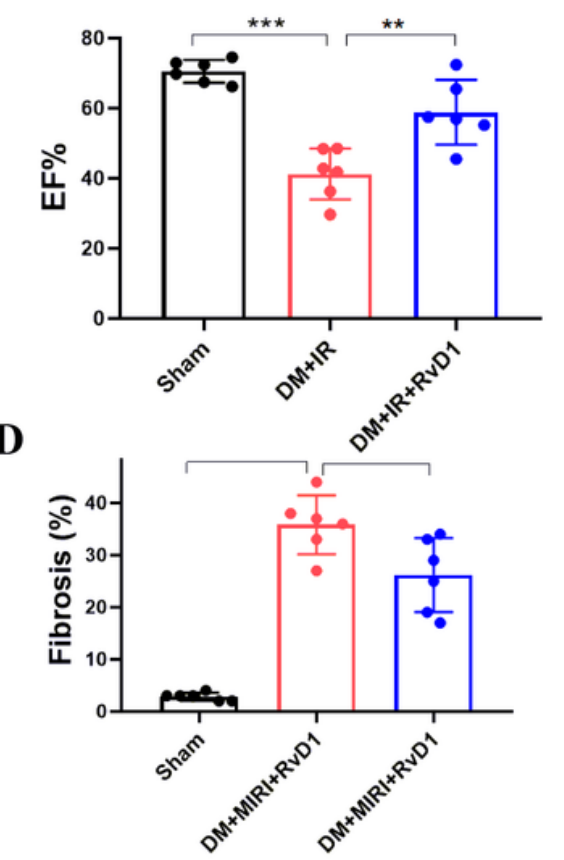

C

$$
\text { Sham }
$$
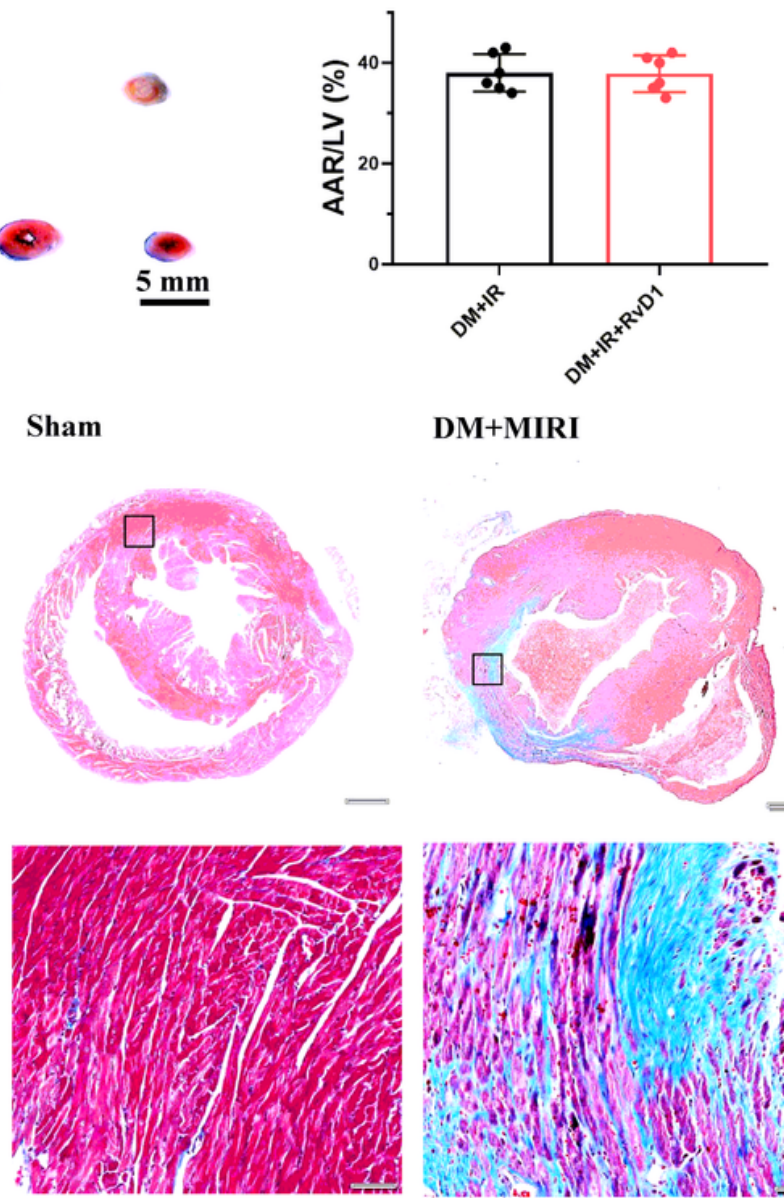

DM+MIRI
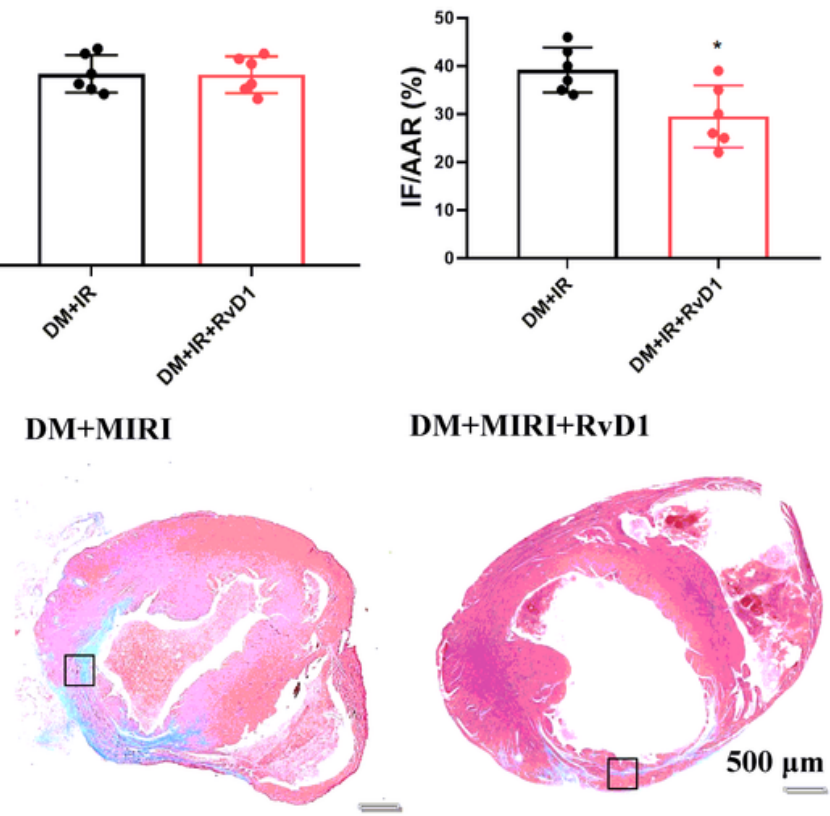

DM+MIRI+RvD1
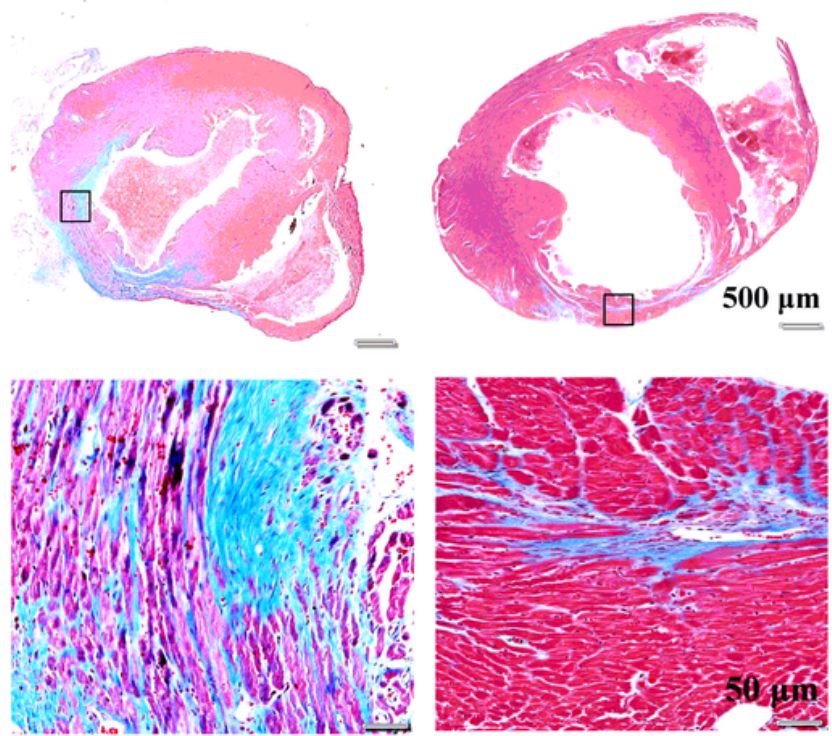

Figure 1

RvD1 preconditioning ameliorated MIRI in diabetic mice A. Representative photographs of Evans blue and TTC staining and quantitative data for infarct size (IF) and area at risk (AAR) in hearts from diabetic mice subjected to MIRI with or without RvD1 pretreatment. B. Echocardiographic analysis at 2 weeks after RvD1 treatment in diabetic MIRI, EF, left ventricular ejection fraction; C. Masson staining for cardiac fibrosis after MIRI in diabetic mice. D. Quantification of cardiac fibrosis in the different groups $(n=6) ;{ }^{*}<<$ $0.05,{ }^{*} \mathrm{p}<0.01,{ }^{* \star *} \mathrm{p}<0.001$. 


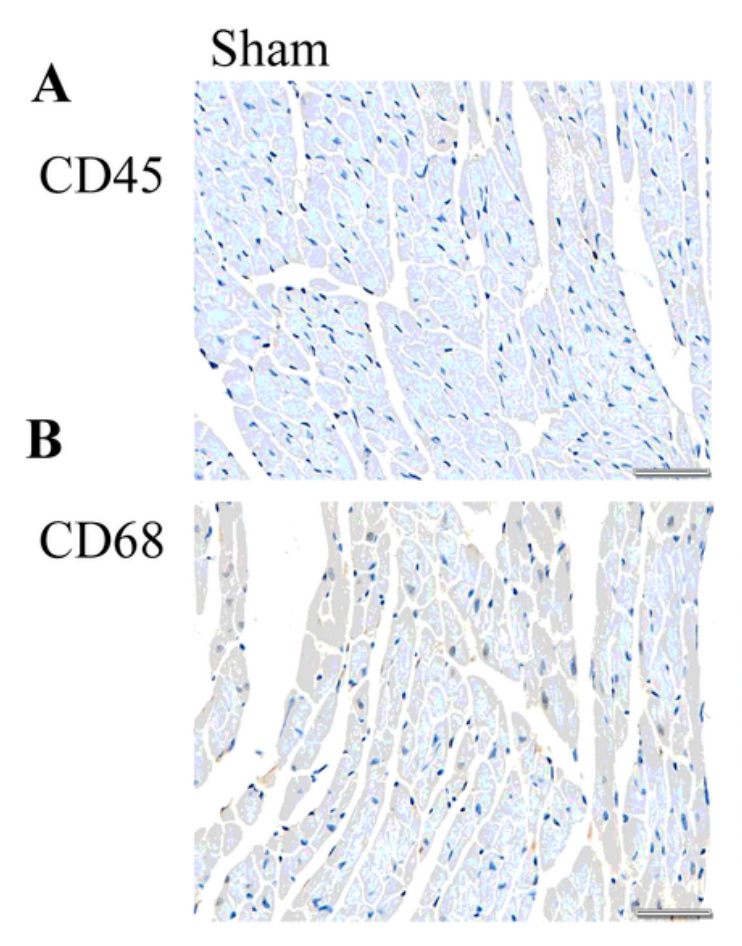

\section{$\mathrm{DM}+\mathrm{MIRI}$}
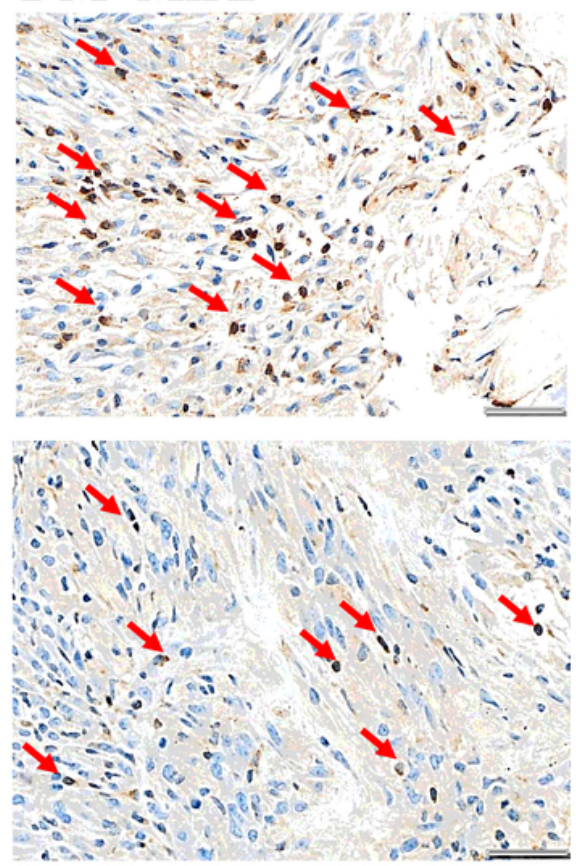

C
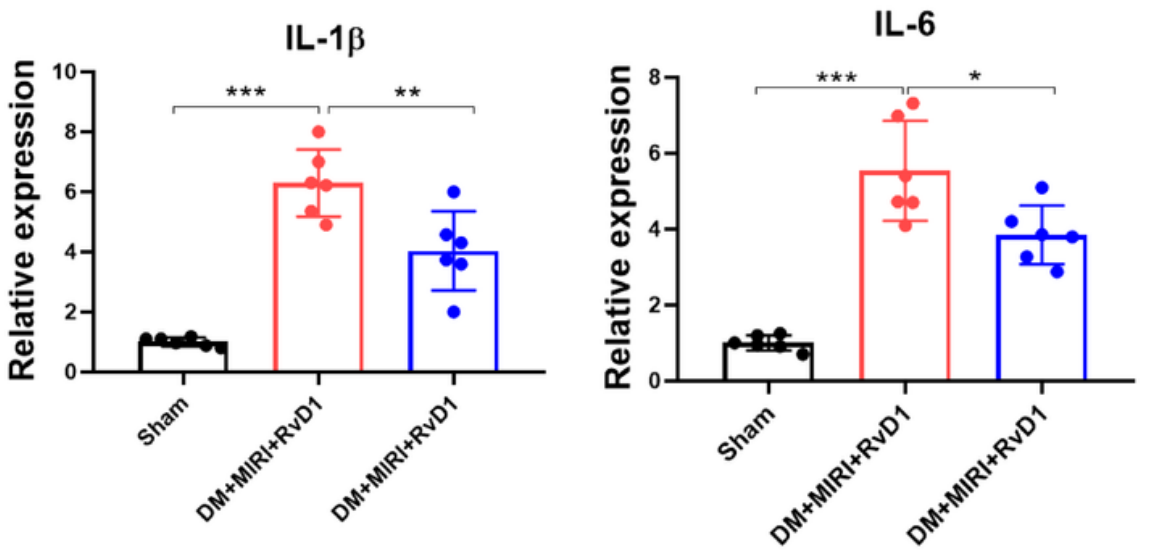

\section{$\mathrm{DM}+\mathrm{MIRI}+\mathrm{RvD}$}
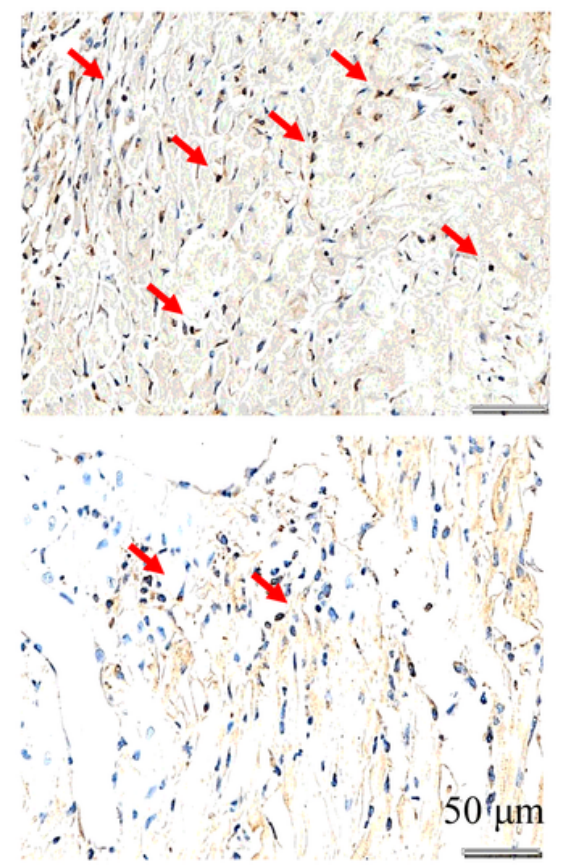

MCP-1

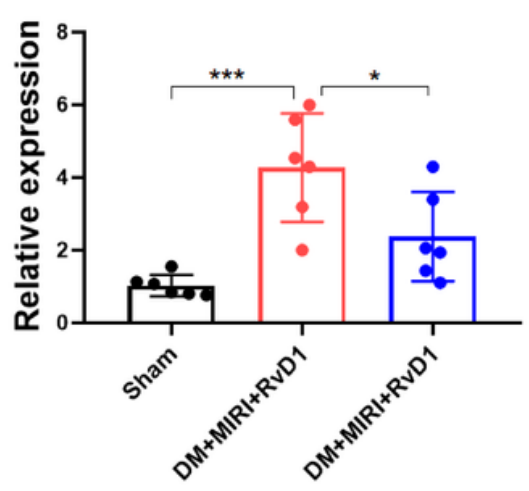

Figure 2

Effect of RvD1 pretreatment on the inflammatory response in the hearts of diabetic mice subjected to MIRI. A. Representative histological images showing CD45+ leukocytes (the red arrows represent CD45positive cells, and blue DAPI staining indicates nuclei) in the hearts of mice in each group. B. Representative histological images showing CD68-positive macrophages in the hearts of mice in each group. C. Changes in the gene expression of inflammatory cytokines in the ischemic myocardium

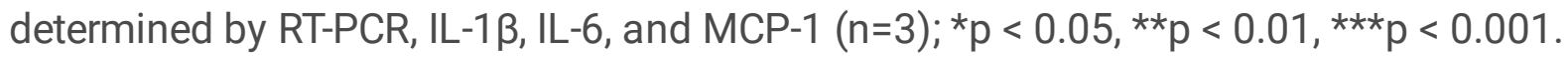




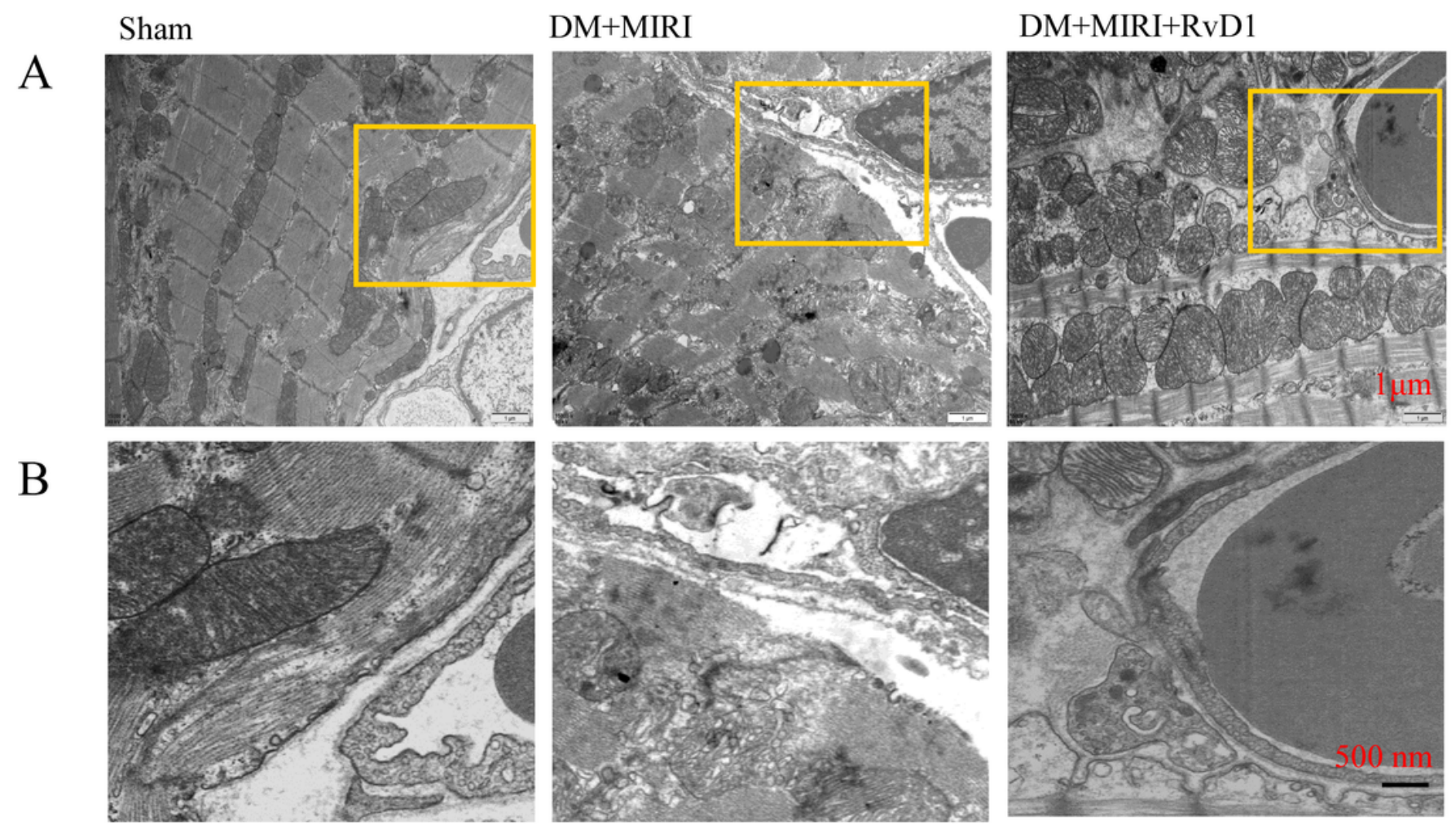

\section{Figure 3}

RvD1 pretreatment reduced MIRI mediated microvascular damage in diabetes. A-B. Representative TEM images of microvascular structures in the hearts of each group after MIRI. The yellow squares indicate the damaged vascular wall, and 4 images were obtained from one heart $(n=3)$.

A DM+MIRI
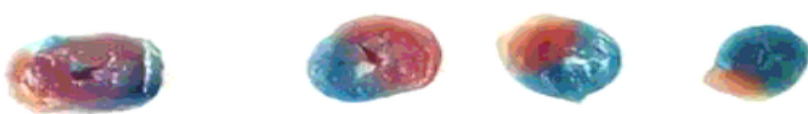

DM+MIRI+RvD1
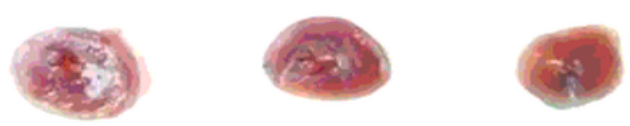
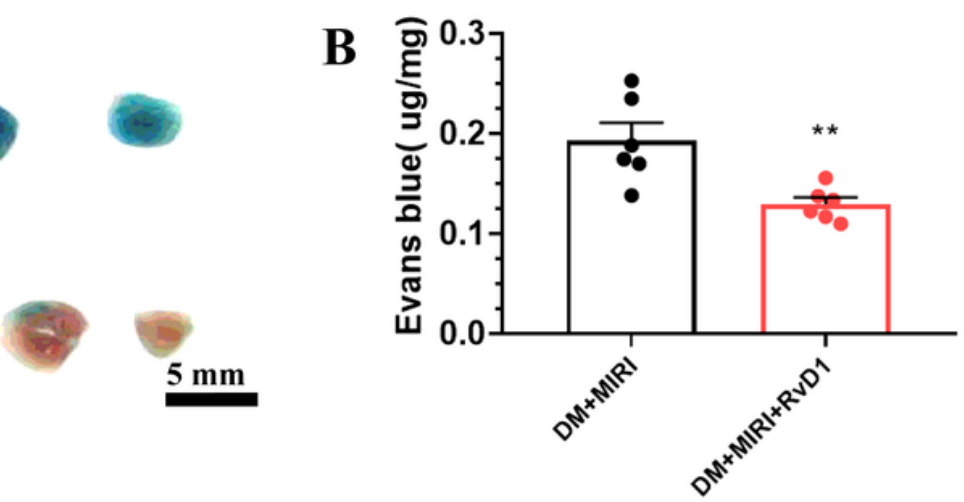

Figure 4

RvD1 reduced endothelial cell permeability after MIRI in diabetes. A. Representative images for Evans blue staining. B. Amount of Evans blue dye extracted from diabetic MIRI hearts in the presence or absence of RvD1 pre-treatment $(n=6) ;{ }^{\star} p<0.05,{ }^{* \star} p<0.01,{ }^{\star *} \mathrm{p} p<0.001$. 
A
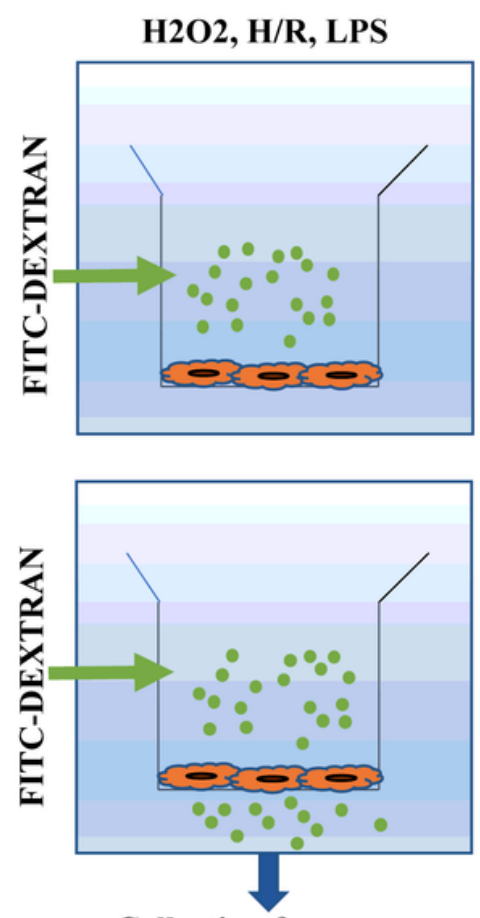

Collection for assay

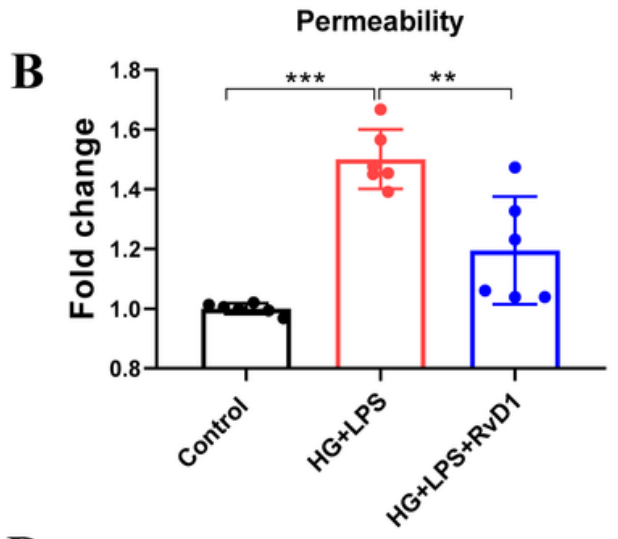

D

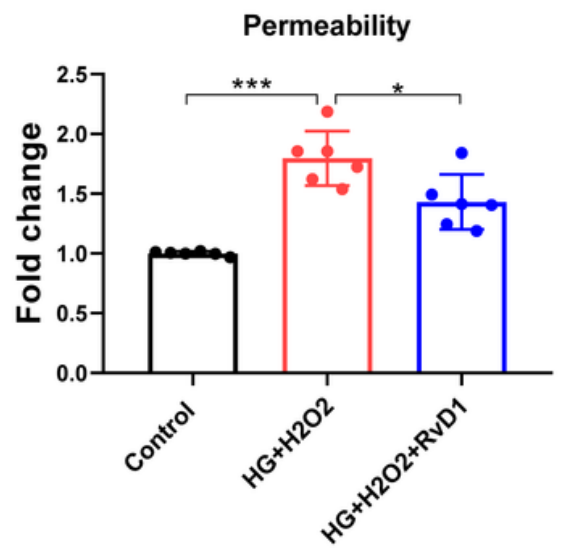

C

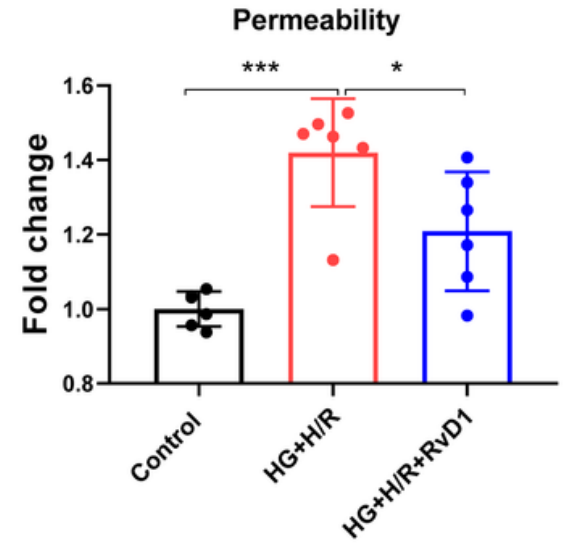

\section{Figure 5}

RvD1 reduced endothelial cell permeability in vitro. A. Transwell device to detected monolayer of HCMECs permeability. B-C. Permeability of the endothelial monolayer stimulated with LPS (400 ng/ml) for 12 hours, hypoxia for 12 hours and reoxygenation for 12 hours and hydrogen peroxide ( $\mathrm{H} 2 \mathrm{O} 2,400 \mu \mathrm{M})$. Changes in permeability were detected by measuring the fluorescence of FITC-dextran $(n=6) ;{ }^{*} p<0.05$, $* \star p$ $<0.01,{ }^{* \star *} p<0.001$.

\section{Figure 6}

RvD1-mediated microvascular protection was regulated by mitochondrial damage under HG conditions. A. RvD1 decreased H2O2-induced mitochondrial reactive oxygen species (MitoROS) in HCMECs under HG conditions, MitoROS determined by MitoSOX Red staining and MitoSOX relative expression analysis using a Celigo imaging cytometer $(n=4)$. B. MitoSOX Red staining images for each group. C. JC-1 analysis of mitochondrial transmembrane potential in HCMECs as determined by flow cytometry analysis, and JC-1 analysis was used to determine the green/red fluorescence ratio $(n=3) ;{ }^{*}<<0.05$, $* \star p$ $<0.01, * \star * p<0.001$. 
A

Control

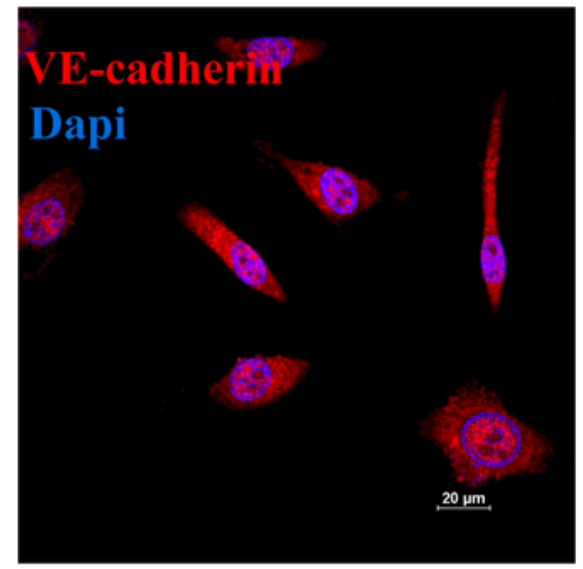

HG+H/R

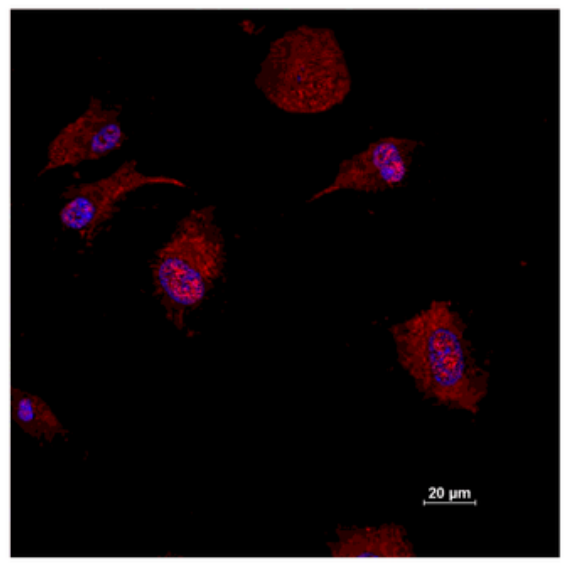

HG+H/R+RvD1

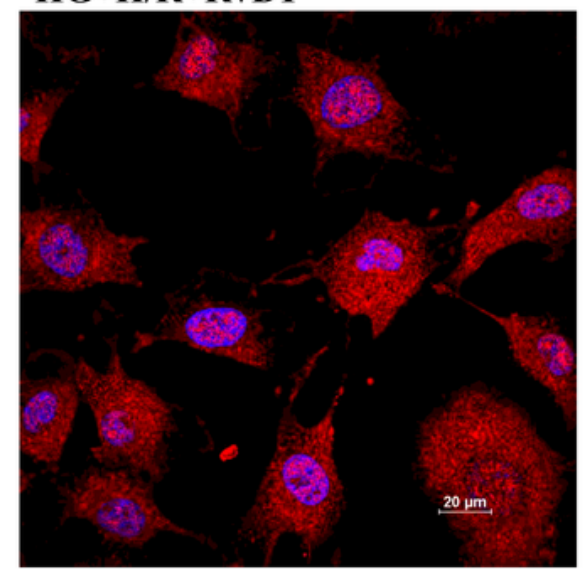

B

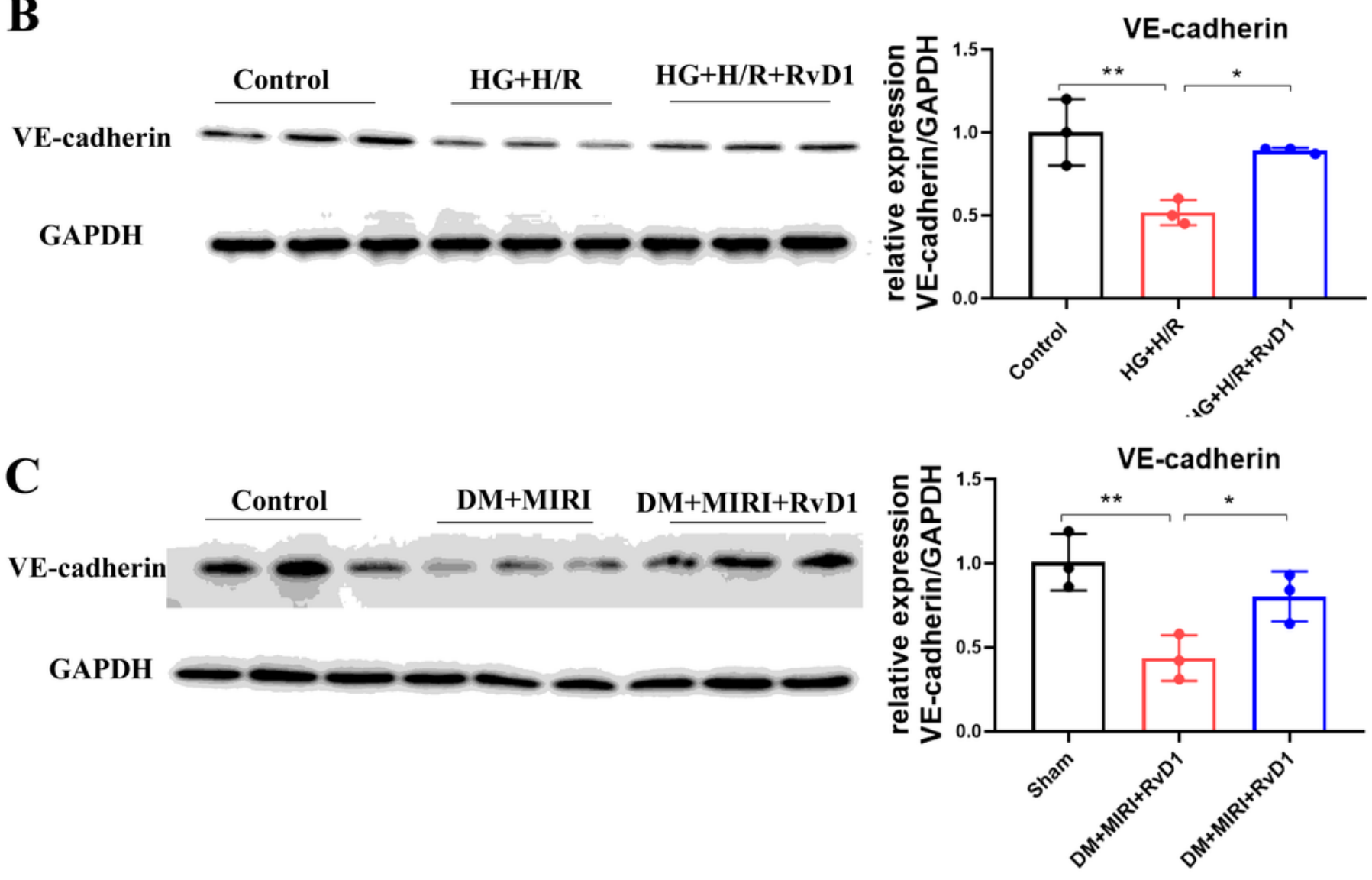

Figure 7

RvD1 decreased endothelial permeability with preservation of VE-cadherin under HG conditions. A. VEcadherin immunostaining in H/R-induced HCMECs with or without RvD1 under HG conditions. B. western blots showing VE-cadherin expression in HG-cultured HCMECs. After $12 \mathrm{~h}$ of hypoxia and $12 \mathrm{~h}$ of reoxygenation, VE-cadherin was reduced, which was reversed by RvD1, and GAPDH was used as a loading control. C. Western blots showing VE-cadherin expression in diabetic MIRI hearts. RvD1 treatment 
increased VE-cadherin expression. Quantification data of myocardial VE-cadherin expression are shown in the plots $(n=3) ;{ }^{*}<<0.05,{ }^{* *} p<0.01, * \star * p<0.001$. 